\title{
Responses of Potato (Solanum tuberosum L.) to Green Manure Cover Crops and Nitrogen Fertilization Rates
}

\author{
Mehmet Sincik • Z. Metin Turan • A. Tanju Göksoy
}

Published online: 5 September 2008

(C) Potato Association of America 2008

\begin{abstract}
This study compared the effects of common vetch (Vicia sativa L.), faba bean (Vicia faba L.), and winter wheat (Triticum aestivum L.; control) cover crop green manures in combination with four rates of nitrogen $(\mathrm{N})$ on tuber yield and quality of three potato varieties (Marfona, Hermes and Agria) during 2-years (2004-2005). Results over years were combined for final analysis. Above-ground dry matter for common vetch and faba bean green manure crops were 2,300 and 2,587 $\mathrm{kg} \mathrm{ha}^{-1}$, respectively. Mean root dry matter yield of faba bean $\left(285 \mathrm{~kg} \mathrm{ha}^{-1}\right)$ was higher than common vetch $\left(120 \mathrm{~kg} \mathrm{ha}^{-1}\right)$. Nitrogen $(\mathrm{N})$ yields of common vetch and faba bean green manure crops were 49 and $58 \mathrm{~kg} \mathrm{Nha}^{-1}$ for above-ground and 2.0 and $5.0 \mathrm{~kg} \mathrm{~N} \mathrm{ha}^{-1}$ for root dry matter, respectively. Averaged over all nitrogen fertilizer rates, potato plants grown in plots following common vetch and faba bean green manure cover crops produced $12.7 \%$ and $15.0 \%$ more tuber yield $\mathrm{ha}^{-1}$, respectively, compared with plants in the winter wheat plots. Potatoes following green manure legume cover crops produced approximately 36 to $38 \%$ higher tuber yields compared with potatoes grown following winter wheat when no nitrogen fertilizer was applied. Differences between tubers yields following cover crops decreased as $\mathrm{N}$ rates increased. Results over combined 2-years indicated
\end{abstract}

The online version of the original article can be found at http://dx.doi. org/10.1007/s12230-008-9011-9

\footnotetext{
M. Sincik $(\varangle) \cdot$ Z. M. Turan • A. T. Göksoy

Faculty of Agriculture, Department of Field Crops,

Uludag University, 16059 Bursa, Turkey

e-mail:sincik@uludag.edu.tr

Z. M. Turan

e-mail: mturan@uludag.edu.tr

A. T. Göksoy

e-mail: agoksoy@uludag.edu.tr
}

that the economically optimum $\mathrm{N}$ rate (EONR) was an average of $30 \mathrm{~kg} \mathrm{~N} \mathrm{ha}^{-1}$ lower for potato following green manure legume cover crops compared with potato following winter wheat cover crop.

Resumen Este estudio comparó los efectos de cultivos de cobertura para abono verde de la arveja común (Vicia sativa L.), haba (Vicia faba L.) y como testigo el trigo de invierno (Triticum aestivum $\mathrm{L}$.), en combinación con cuatro dosis de nitrógeno $(\mathrm{N})$, sobre los rendimientos y calidad de tubérculos de tres variedades de papa (Marfona, Hermes y Agria), durante dos años (2004-2005). Los resultados de años fueron combinados para el análisis final. La materia seca del follaje para los cultivos de arveja y haba para abono verde fue de 2300 y $2587 \mathrm{Kg} \mathrm{ha}^{-1}$, respectivamente. En promedio el rendimiento de materia seca de la raíz de haba $\left(285 \mathrm{~kg} \mathrm{ha}^{-1}\right)$ fue más alta que la de arveja común (120 kg. de $\mathrm{N} \mathrm{ha}^{-1}$ ). Los rendimientos de $\mathrm{N}$ de los cultivos de arveja común y haba para abono verde fueron de $48 \mathrm{y}$ $59 \mathrm{~kg}$. de $\mathrm{N} \mathrm{ha}^{-1}$ para el follaje y 2.0 y $5.0 \mathrm{~kg}$. $\mathrm{N} \mathrm{ha}^{-1}$ para la materia seca de la raíz, respectivamente. En promedio sobre todas las dosis de abono nitrogenado, las plantas de papa cultivadas en parcelas a continuación de los cultivos de cobertura de arveja y haba para abono verde produjeron $12.7 \%$ y $15.0 \%$ más de rendimiento de tubérculos por ha ${ }^{-1}$, respectivamente, comparados con plantas que crecieron en las parcelas de trigo de invierno. Papa después de cultivos de cobertura de leguminosas para abono verde produjo aproximadamente 36 a $38 \%$ mas de rendimiento de tubérculos en comparación con papa cultivada después de trigo de invierno cuando no se aplicó fertilizante nitrogenado. Las diferencias en el rendimiento de tubérculos a continuación de cultivos de cobertura disminuyó a medida que la dosis de $\mathrm{N}$ se incrementó. Los resultados combinados de dos años indican que el dosis de $\mathrm{N}$ económicamente óptima 
(EONR) fue en promedio de $30^{\mathrm{kg} \text { de } \mathrm{N} \text { ha-1 }}$ mas bajo para papa a continuación de cultivos de cobertura de leguminosas para abono verde comparado con papa a continuación de cultivo de cobertura de trigo de invierno.
The Resumen was inadvertently omitted from this article which was published in vol. 85 no. 2 , p. 150 . It is reproduced here along with the English abstract.

Springer regrets the error. 